\title{
OPEN Demystifying the catalytic pathway of Mycobacterium tuberculosis isocitrate lyase
}

\author{
Collins U. Ibeji ${ }^{1,2,3}$, Nor Amirah Mohd Salleh ${ }^{1}$, Jia Siang Sum ${ }^{1}$, Angela Chiew Wen Ch'ng ${ }^{1}$, \\ Theam Soon Lim ${ }^{1} \&$ Yee Siew Choong ${ }^{1 凶}$
}

Pulmonary tuberculosis, caused by Mycobacterium tuberculosis, is one of the most persistent diseases leading to death in humans. As one of the key targets during the latent/dormant stage of $M$. tuberculosis, isocitrate lyase (ICL) has been a subject of interest for new tuberculosis therapeutics. In this work, the cleavage of the isocitrate by $M$. tuberculosis ICL was studied using quantum mechanics/ molecular mechanics method at M06-2X/6-31+G(d,p): AMBER level of theory. The electronic embedding approach was applied to provide a better depiction of electrostatic interactions between MM and QM regions. Two possible pathways (pathway I that involves Asp108 and pathway II that involves Glu182) that could lead to the metabolism of isocitrate was studied in this study. The results suggested that the core residues involved in isocitrate catalytic cleavage mechanism are Asp108, Cys191 and Arg228. A water molecule bonded to $\mathrm{Mg}^{2+}$ acts as the catalytic base for the deprotonation of isocitrate $\mathrm{C}(2)-\mathrm{OH}$ group, while Cys191 acts as the catalytic acid. Our observation suggests that the shuttle proton from isocitrate hydroxyl group C (2) atom is favourably transferred to Asp108 instead of Glu182 with a lower activation energy of $6.2 \mathrm{kcal} / \mathrm{mol}$. Natural bond analysis also demonstrated that pathway I involving the transfer of proton to Asp108 has a higher intermolecular interaction and charge transfer that were associated with higher stabilization energy. The OM/MM transition state stepwise catalytic mechanism of ICL agrees with the in vitro enzymatic assay whereby Asp108Ala and Cys191Ser ICL mutants lost their isocitrate cleavage activities.

Isocitrate lyase (ICL) plays an important role in the metabolic processes of citric, methylcitric and glyoxylate cycles $^{1,2}$. During the latent/dormant stage, Mycobacterium tuberculosis ICL converts isocitrate to glyoxylate and succinate for energy generation via the glyoxylate cycle ${ }^{3}$. Studies also showed that $M$. tuberculosis cannot survive in a latent TB model without ICL ${ }^{4-6}$. Therefore, ICL has become one of the major targets for latent M. tuberculosis. The structure of M. tuberculosis ICL in complex with the isocitrate metabolites (glyoxylate and succinate) that was solved in year $2000^{7}$ has enabled the study of $\mathrm{ICL}^{8-11}$, as well as to search for possible inhibitors for ICL ${ }^{12-14}$.

The inhibition mechanism of the ICL family has been proposed from experimental finding $\mathrm{s}^{15-18}$. However, the actual process of isocitrate cleavage/metabolism remains unclear. The mechanism of isocitrate cleavage was reported to be involved in the deprotonation of isocitrate $\mathrm{C}(2)-\mathrm{OH}$ group which resulted in the cleavage of the $C(2)-C(3)$ bond trailed by the protonation of the carbanion ${ }^{15,19}$ that progresses towards the $C(3)$ position. Two mechanisms have been proposed for the cleavage of isocitrate. The first proposed mechanism stated that two water molecules adjacent to $\mathrm{Mg}^{2+}$ may be involved in the deprotonation of isocitrate $\mathrm{C}(2)-\mathrm{OH}$ group that leads to the formation of an anionic intermediate ${ }^{15}$. The orientation and specific distances from the substrate hydroxyl group $(\mathrm{C}(2)-\mathrm{OH})$ also suggested two water molecules as the candidates to act as the catalytic base (removing protons; one close to Asp108 and the other to Glu182). The deprotonations from $\mathrm{C}(2)-\mathrm{OH}$ is then followed by the transfer of proton to either Asp108 (Asp58 of 2-methylisocitrate lyase, MICL) or Glu182 (Glu115 of MICL). Cys191 or Asp87 will then act as the catalytic acid in the protonation of the C(3) atom of the cleaved isocitrate. The catalytic acid (also known as general acid) is referred to an amino acid that participates in the mechanism of hydrolysis responsible for protonating (adding a proton) the succinate leaving group in the ICL cleavage mechanism. The second proposed mechanism involved Tyr43 as a catalytic base in the transfer of proton to His114 bonded to water molecules, resulting in the shuttling of a proton from isocitrate $\mathrm{C}(2)-\mathrm{OH}$

${ }^{1}$ Institute for Research in Molecular Medicine (INFORMM), Universiti Sains Malaysia, 11800 Minden, Penang, Malaysia. ${ }^{2}$ Catalysis and Peptide Research Unit, School of Health Sciences, University of KwaZulu-Natal, Durban 4041, South Africa. ${ }^{3}$ Department of Pure and Industrial Chemistry, Faculty of Physical Sciences, University of Nigeria, Nsukka 410001, Enugu State, Nigeria. ${ }^{\boxplus}$ email: yeesiew@usm.my 


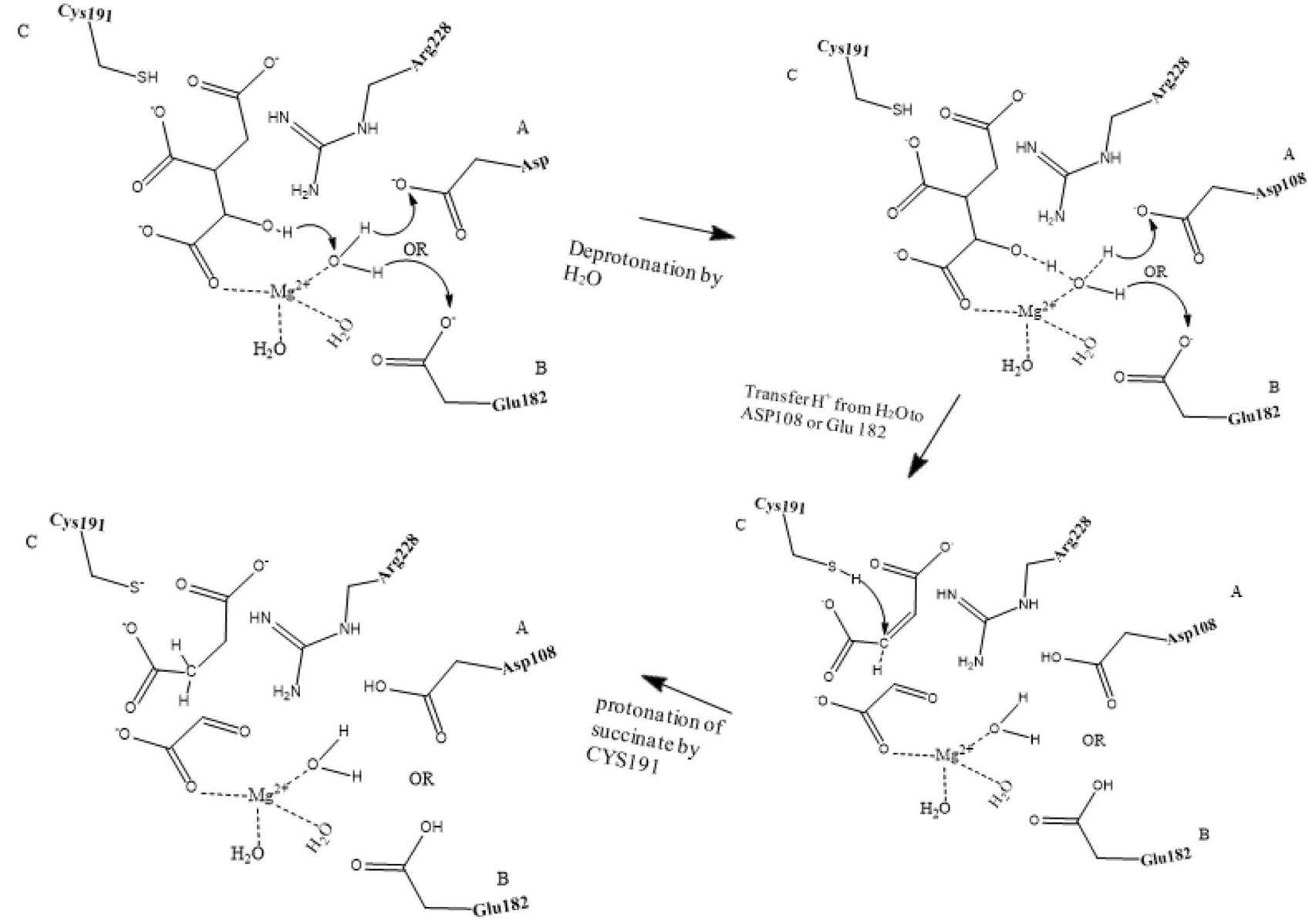

Figure 1. Possible catalytic mechanism of $M$. tuberculosis isocitrate lyase (ICL) for an acid/base-catalyzed conversion of substrate (isocitrate) to metabolites (glyoxylate and succinate). Step 1: Transfer of proton to either (A) Asp108 or (B) Glu182; Step 2: Proton transfer from Cys191 SH group to succinate C(3).

group to Glu115 ${ }^{17,18}$. Besides, Arg161 and Tyr89 of MICL (Arg 228 and Tyr43 in ICL) were also proposed as the unidentified catalytic base $\mathrm{b}^{16,20}$.

The hybrid quantum mechanics/molecular mechanics (QM/MM) approach has been used to gain insight into the reaction mechanism of enzymes ${ }^{21-23}$. The QM/MM approach has also been applied to understand the catalytic mechanisms for lyases such as aspartate ammonia lyase ${ }^{24}, \mathrm{~N}$-acetylneuraminic acid lyase $\mathrm{a}^{25}, \alpha$-1,4-glucan lyase $\mathrm{e}^{26}$, (R)-hydroxynitrile lyase $\mathrm{e}^{27}$ and 2,3-dimethylmalate lyase (DMML) ${ }^{16}$. Studies on IMP dehydrogenase, pectate/pectin lyases, fumarate reductase, and L-aspartate oxidase ${ }^{28}$, as well as citrate synthase ${ }^{29}$ suggested that Arg could be the catalytic base while calculations on $\mathrm{DMML}^{16}$ and pyruvate formate-lyase ${ }^{30}$ proposed that Cys as the catalytic acid. However, the identity of the catalytic base involved in the deprotonation of $\mathrm{C}(2)-\mathrm{OH}$ is yet to be resolved.

Herein, we applied QM/MM (ONIOM) approach to gain a better understanding on the mechanism involved in the breaking of isocitrate into glyoxylate and succinate. We explored the two proposed pathways by reported experimental works and identified the probable acidic and basic residues involved in the mechanistic process. This, in turn could be useful in providing computational models for the design of new anti-TB drugs.

\section{Results}

The catalytic mechanism associated with the breakdown of isocitrate by M. tuberculosis ICL (Fig. 1) was explored using QM/MM (ONIOM) approach. In this work, we studied two different pathways (namely pathway I and II) that could lead to the metabolism of isocitrate (Figs. 1,2). These pathways would involve two residues, one as the catalytic acid and the other as the catalytic base as observed experimentally ${ }^{15}$. The relative activation energies associated with the catalytic mechanism for pathways I and II are presented in Table 1 and Fig. 3. Single-point calculations with two additional density functionals (mPWB1W and $\omega \mathrm{b} 97 \mathrm{XD}$ ) paired with 6-311++G (2d, 2p) in conjunction with electronic embedding were employed to explore the sensitivity of the applied functionals. These functionals have been reported to give accurate energetics for kinetics and thermodynamics ${ }^{31}$. The twodimensional (2D) PES was performed to visualise the cleavage mechanism. The scan result suggested that the cleavage mechanism is a stepwise mechanism (Fig. 4). The figure showed that a concerted catalytic mechanism is unlikely since the deprotonation of isocitrate $\mathrm{C}(2)-\mathrm{OH}$ and a proton transfer to the acceptor residue occurs before the elongation of the $\mathrm{C}(2)-\mathrm{C}(3)$ bond and thereafter leading to bond breaking. This is consistent with the 

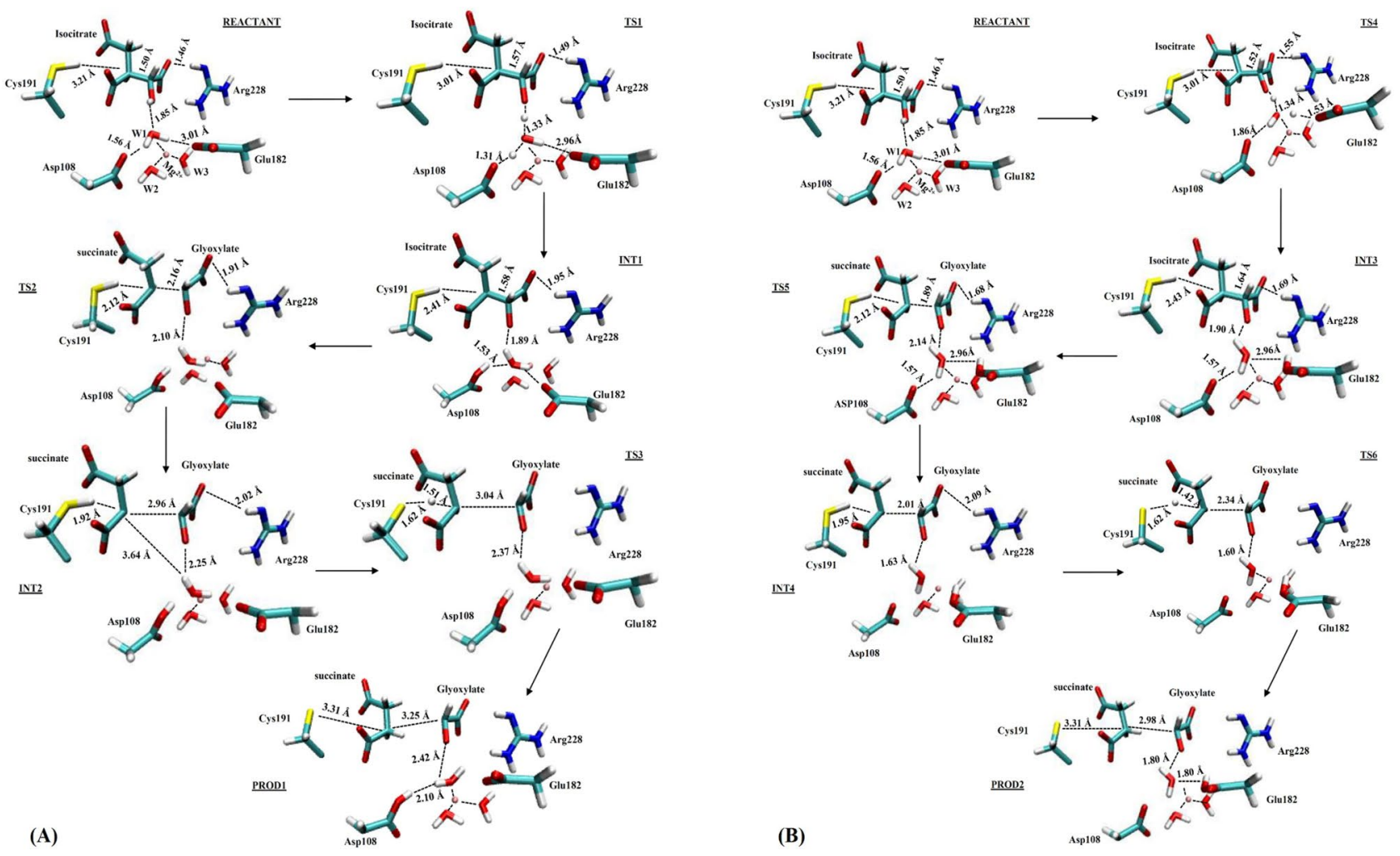

Figure 2. Graphical diagram of QM/MM stationary points at the M06-2X/6-31+G(d,p)/AMBER level of theory for possible (A) pathway I and, (B) pathway II, in the cleavage of isocitrate by $M$. tuberculosis isocitrate lyase (ICL).

\begin{tabular}{|c|c|c|c|c|c|c|}
\hline \multirow[b]{2}{*}{ Reaction path } & \multicolumn{2}{|c|}{ M06-2X $^{a}$} & \multicolumn{2}{|c|}{ mPWB1W ${ }^{b}$} & \multicolumn{2}{|c|}{$\omega b 97 X D^{c}$} \\
\hline & $\Delta \mathbf{E}$ & $\Delta \mathbf{G}^{*}$ & $\Delta \mathbf{E}$ & $\Delta G^{*}$ & $\Delta \mathbf{E}$ & $\Delta \mathrm{G}^{*}$ \\
\hline \multicolumn{7}{|l|}{ Pathway I } \\
\hline $\mathrm{R}$ & 0.00 & 0.00 & 0.00 & 0.00 & 0.00 & 0.00 \\
\hline TS1 & 6.2 & 6.1 & 6.1 & 6.0 & 6.2 & 6.1 \\
\hline INT1 & 3.8 & 3.0 & 3.6 & 2.9 & 3.9 & 3.1 \\
\hline TS2 & 9.1 & 8.6 & 9.2 & 8.4 & 9.7 & 8.8 \\
\hline INT2 & 9.4 & 9.2 & 9.2 & 9.0 & 9.5 & 9.3 \\
\hline TS3 & 11.1 & 10.0 & 11.0 & 9.9 & 11.8 & 10.1 \\
\hline PROD1 & -2.1 & -3.4 & -2.2 & -3.5 & -2.9 & 3.8 \\
\hline \multicolumn{7}{|l|}{ Pathway II } \\
\hline TS4 & 32.3 & 32.0 & 32.4 & 32.2 & 32.5 & 32.4 \\
\hline INT3 & 17.6 & 17.8 & 17.9 & 17.8 & 18.0 & 17.9 \\
\hline TS5 & 33.1 & 35.2 & 35.8 & 35.4 & 35.9 & 35.7 \\
\hline INT4 & 30.1 & 29.8 & 30.7 & 30.3 & 30.6 & 30.6 \\
\hline TS6 & 34.5 & 34.2 & 34.6 & 34.4 & 34.7 & 34.6 \\
\hline PROD2 & 5.3 & 4.1 & 4.5 & 4.2 & 4.6 & 4.1 \\
\hline
\end{tabular}

Table 1. Reaction mechanism (relative) energy ( $\mathrm{kcal} / \mathrm{mol}$ ) obtained from ONIOM using different density functionals at 6-311++G(2d,2p): Amber for the breakdown of isocitrate by $M$. tuberculosis isocitrate lyase (ICL). ${ }^{a, b, c}$ Relative (to reactant) total electronic energy $(\Delta \mathrm{E})$ and activation free energy $\left(\Delta \mathrm{G}^{\ddagger}\right.$, with thermal correction) at B3LYP, M06-2X, $\omega$ B97X/6-311++G(d,p):AMBER//B3LYP/6-31G(d,p):AMBER level. $R$ reactant, TS transition state, INT intermediate, $P R O D$ product.

observation for the mechanism of DMML, a subclass of the lyase family ${ }^{16}$. The starting structure for the nonconstrained transition state was obtained from the scan coordinate (Fig. 4).

Reaction mechanism for pathway I. The QM/MM reactant structure obtained from the restrained $\mathrm{MD}$ simulation is similar to the X-ray crystal structure with comparable key atomic distances (Supplementary 


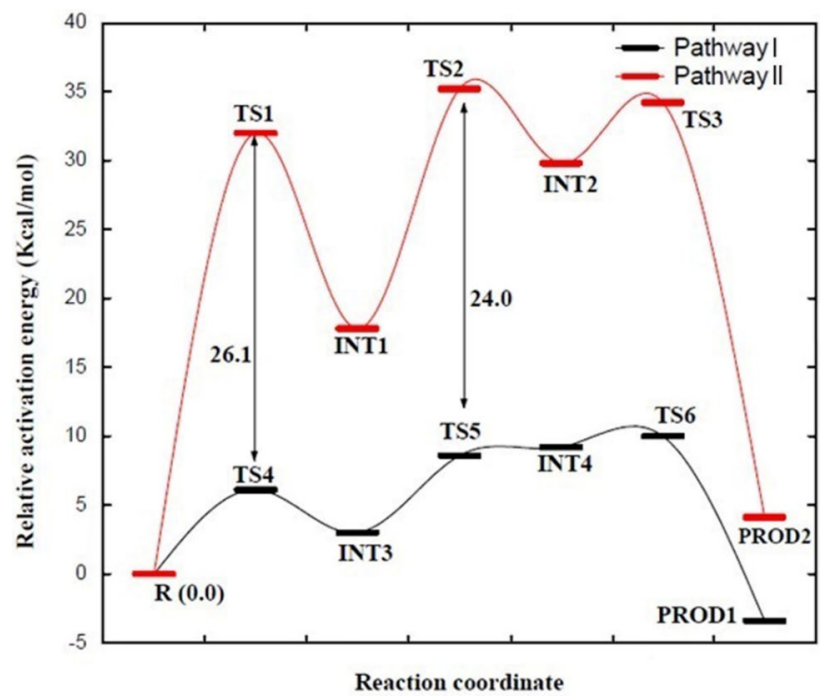

Figure 3. Relative Gibb free energy profile at (ONIOM) M06-2X/6-311++G(2d,2p) for the stepwise cleavage mechanism of isocitrate by M. tuberculosis isocitrate lyase (ICL).
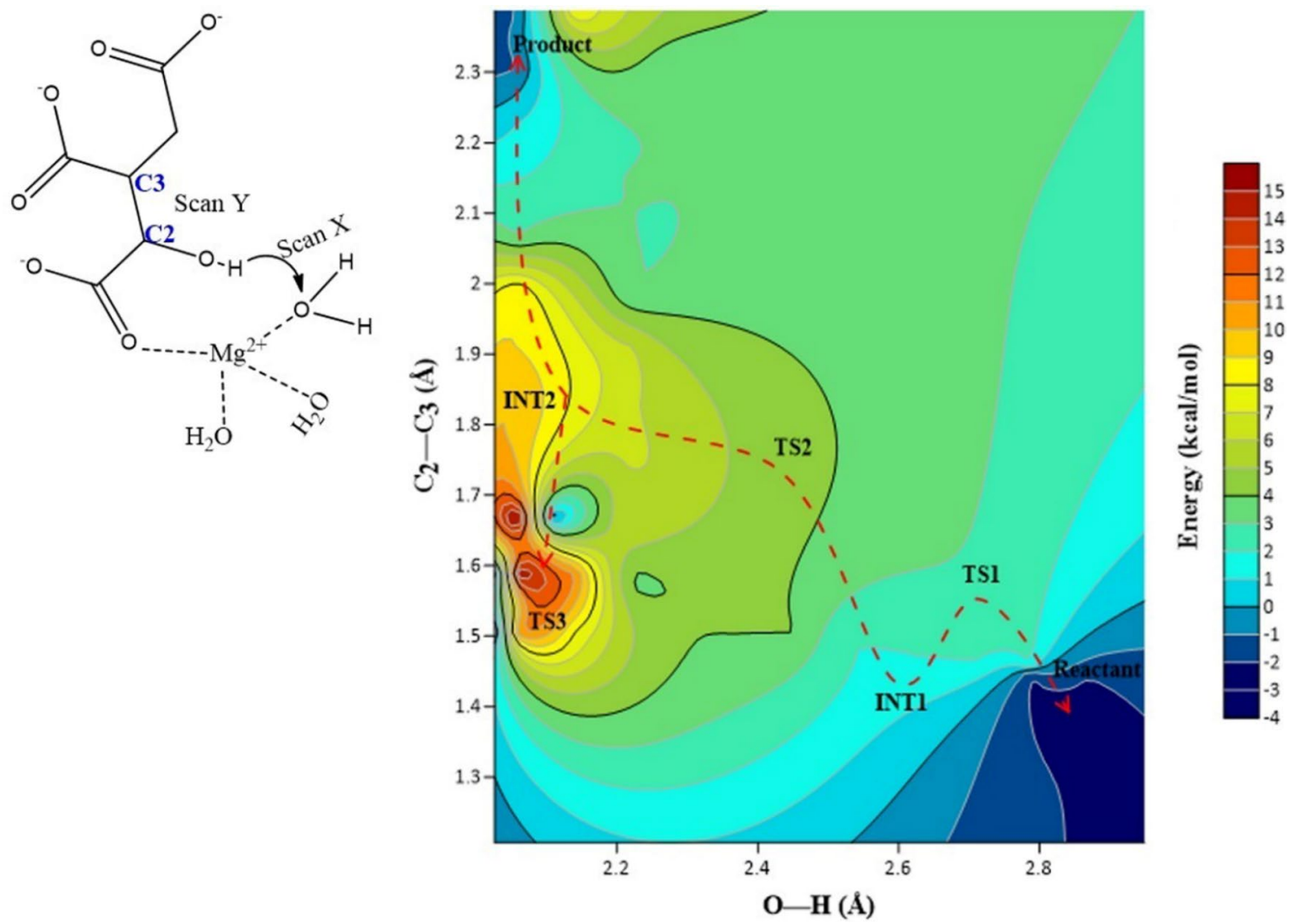

Figure 4. $2 \mathrm{D}$ potential energy contour map showing a stepwise cleavage mechanism of isocitrate by $M$. tuberculosis isocitrate lyase (ICL) obtained from an ONIOM M06-2X/6-31+G(d,p)/AMBER scan calculation. TS is the transition state while INT is the intermediate.

Information Figure S1). The RMSD plot from the MD simulation is presented in Supplementary Information Figure S2. The ICL active site consists of a metal-dependent $\mathrm{Mg}^{2+}$ cofactor coordinated to three water molecules (namely W1, W2 and W3) with interatomic distances $\left(\mathrm{Mg}^{2+}-\mathrm{O}\right)$ of $1.96,2.01$ and $2.16 \AA$, respectively. It is also evidenced from the structural data that the substrate (isocitrate) for ICL is activated for deprotonation through the binding of $\mathrm{Mg}^{2+}$ to isocitrate $\mathrm{C}(2)-\mathrm{O}$ carboxyl atom. The substrate interacts strongly with $\operatorname{Arg} 228$ with a short hydrogen bond distance of $1.46 \AA$ corresponding to O-H atom (Fig. 2A). As suggested by X-ray experi- 


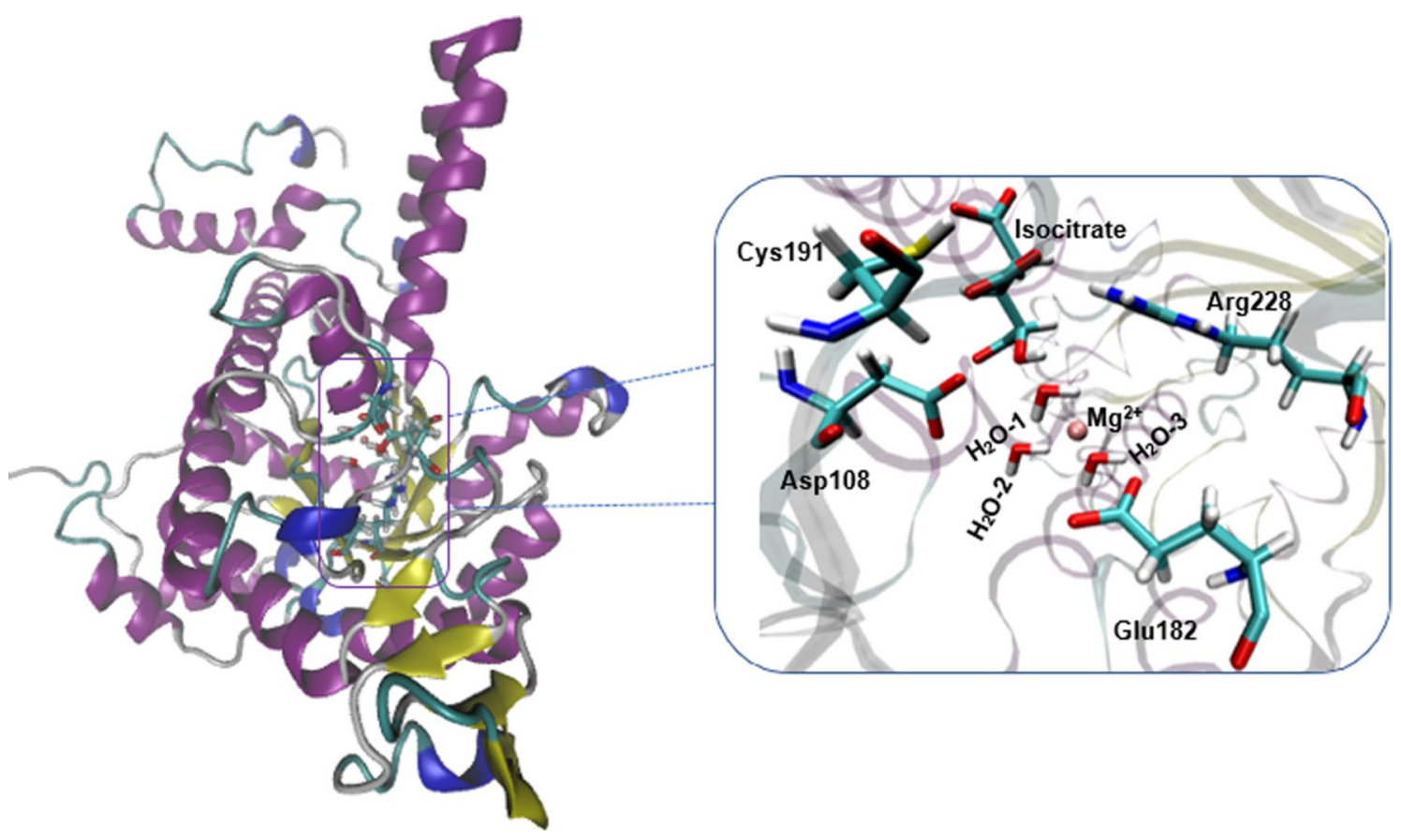

Figure 5. 3D structural depiction of the QM/MM ONIOM model in the study of $M$. tuberculosis isocitrate lyase (ICL) catalytic pathway. The optimized the geometries of the high layer $(\mathrm{QM})$ at the B3LYP/6-31+G(d,p) is in stick presentation while outside the box is the MM region in ribbon presentation.

ment data ${ }^{15}$, two possible water molecules may mediate the deprotonation of isocitrate $\mathrm{C}(2)-\mathrm{OH}$ based on the orientation and distance to the $\mathrm{C}(2)-\mathrm{OH}$ group of isocitrate. FigureS 1 and $2 \mathrm{~A}$ show that although water molecules W1 and W2 are coordinated to $\mathrm{Mg}^{2+}$, the position of W1 is closer and oriented to transfer the proton from isocitrate $\mathrm{C}(2)-\mathrm{OH}$ with the interatomic distance of $1.85 \AA$. The proposed catalytic base identified as Cys 191 is close enough to isocitrate $\mathrm{C}(3)$ with an interatomic distance of 3.21 $\AA$. Further details on the optimized geometries of the high layer (QM) ONIOM model and the redundant coordinate of isocitrate lyase can be found in the Supplementary Information Figure S3.

The function of water molecule (W1) in pathway I. It has been previously reported that water may act as a catalytic base in the cleavage mechanisms ${ }^{15}$. As shown in Fig. 1, a catalytic base is responsible for the isocitrate activation via proton abstraction. It was observed from the crystal structure ${ }^{15}$ that there are three water molecules (W1, W2, W3) coordinated to $\mathrm{Mg}^{2+}$ (Figs. 4, 5). These water molecules are oriented towards the hydroxyl group attached to isocitrate $\mathrm{C}(2)$ atom with W1 having the shortest distance of $1.85 \AA$, that is close enough to cause deprotonation of the isocitrate $\mathrm{OH}$ group. The energetics of the reaction step were studied and a concerted process was observed for the deprotonation of the hydroxyl group attached to isocitrate $\mathrm{C}(2)$ atom with concurrent deprotonation of W1 (Fig. 2A). The relative Gibbs free energy of activation $\left(\Delta \mathrm{G}^{\ddagger}\right)$ associated with this state (TS1) was observed to be $6.1 \mathrm{kcal} / \mathrm{mol}$ (Table 1). An intermediate (INT1) is formed in the process by the transfer of a proton from W1 to Asp108 with a distance of $1.31 \AA$, similar to the deprotonation distance of isocitrate $\mathrm{C}(2)-\mathrm{OH}$. The $\Delta \mathrm{G}^{\ddagger}$ associated with the intermediate state is about $3 \mathrm{kcal} / \mathrm{mol}$ lesser than TS1. We also evaluated if $\operatorname{Arg} 228$ can function as a catalytic base to deprotonate isocitrate $\mathrm{C}(2)-\mathrm{OH}$. However, the data showed that isocitrate becomes unstable as the orientation changed, resulting in an elongated distance beyond $5 \AA$ A between isocitrate $\mathrm{C}(2)-\mathrm{OH}$ and Arg228. This suggests that W1 is more likely to act as the catalytic base in the mechanism of the reaction.

The function of Asp108 in pathway I. It was proposed that water molecules may shuttle proton from the hydroxyl group of isocitrate C(2) via Asp108 or Glu182 (Fig. 1) $)^{15}$. To demonstrate Asp108 is key to this process, S. enterica Asp108 (Asp58 in MICL) was substituted with Ala and the result showed that Asp108 was essential in the catalytic outcome ${ }^{15}$. The $2 \mathrm{D}$ potential energy surface (PES) showed that the proton from $\mathrm{C}(2)-\mathrm{OH}$ group was transferred to Asp108 (INT1) before the cleavage of the C2-C3 bond (TS2). Asp108 was found to form a perturbed bond with $\mathrm{Mg}^{2+}$ by direct coordination and the energetics for the mechanistic process showed that the transfer of a proton from W1 to Asp108 was preferred and favoured compared to Glu182 (Table 1; Fig. 2B). In this barrierless process, it was also observed that the distance between Cys191 and isocitrate $\mathrm{C}(3)$ has reduced considerably from 3.01 to $2.41 \AA$ A. This may indicate a characteristic hydrogen bonding between the electron-rich isocitrate C(3) atom and Cys191 thiol group. Thus, suggesting that Cys191 could be the catalytic acid in isocitrate cleavage process. Further analysis of the energetics (INT1) using a different functional (mPWB1W) revealed that the process is barrierless with lower $\Delta \mathrm{G}^{\ddagger}$ of $2.9 \mathrm{kcal} / \mathrm{mol}$. 


\begin{tabular}{|c|c|c|}
\hline Donor & Acceptor & $\mathrm{E}^{2}(\mathrm{kcal} / \mathrm{mol})$ \\
\hline \multicolumn{3}{|l|}{ TS1 } \\
\hline $\mathrm{LP}\left(\mathrm{O}_{68}\right)$ & $\sigma^{\star}\left(\mathrm{O}_{85}-\mathrm{H}_{81}\right)$ & 64.4 \\
\hline $\mathrm{LP}\left(\mathrm{O}_{85}\right)$ & $\sigma^{\star}\left(\mathrm{O}_{10}-\mathrm{H}_{86}\right)$ & \begin{tabular}{|l|l|}
78.8 \\
\end{tabular} \\
\hline \multicolumn{3}{|l|}{\begin{tabular}{|l|} 
TS4 \\
\end{tabular}} \\
\hline $\mathrm{LP}\left(\mathrm{O}_{68}\right)$ & $\sigma^{*}\left(\mathrm{O}_{85}-\mathrm{H}_{81}\right)$ & 31.1 \\
\hline $\mathrm{LP}\left(\mathrm{O}_{85}\right)$ & $\sigma^{\star}\left(\mathrm{O}_{24}-\mathrm{H}_{90}\right)$ & 24.6 \\
\hline
\end{tabular}

Table 2. Second-order perturbation stabilization energies ( $\mathrm{kcal} / \mathrm{mol})$ corresponding to the main intermolecular charge transfer interaction (Donor $\rightarrow$ Acceptor) obtained at M06-2X/6-311++g(d,p) for $M$. tuberculosis isocitrate lyase (ICL) for pathway I and II transition states.

The role of Arg228 in pathway I. In DMML, Arg161 (corresponding to Arg228 of M. tuberculosis ICL) was reported to be potentially responsible for the deprotonation of isocitrate $\mathrm{C}(2)-\mathrm{OH}$ which leads to the cleavage of isocitrate $\mathrm{C}(2)-\mathrm{C}(3)$ bond ${ }^{16,20}$. However, our study showed alternative findings as ICL Arg228 is already in the protonated state and could not serve as a proton shuttle from isocitrate $\mathrm{C}(2)-\mathrm{OH}$ that leads to the isocitrate cleavage. But our observation is similar to MICL experimental findings ${ }^{15}$ whereby there is no basis for Arg228 to mediate a proton transfer based on its protonated state since it only aids in intermediate stabilization. In this study, step 3 of the mechanism involves the breaking of isocitrate $\mathrm{C}(2)-\mathrm{C}(3)$ bond and it was observed that Arg228 plays an important role in this step. In the reactant stage, Arg228 forms a strong bond with the carboxylate group of the substrate (Fig. 2A) which stabilizes the substrate and aids the intermediate stabilization. After the deprotonation of the $\mathrm{C}(2)$ atom hydroxy group, Arg228 pulls out from the substrate with an elongated bond distance from $1.49 \AA$ (reactant TS1) to $1.91 \AA$ (TS2) which further resulted in the destabilization of the substrate thus leading to the cleavage of the $\mathrm{C}(2)-\mathrm{C}(3)$ bond (TS2). In this step, the interatomic distance between $\mathrm{C}(2)$ and $\mathrm{C}(3)$ atom changes from 1.50 to $2.16 \AA$ (reactant to TS2).

The function of Cys191 in pathway I. In MICL, Cys123 (corresponding to Cys191 in M. tuberculosis ICL) was found to show significant effect on the stereochemistry and mechanism of the isocitrate $\mathrm{C}(2)-\mathrm{C}(3)$ bond cleavage $\mathrm{e}^{15}$. The cleavage of isocitrate resulted in the formation of a carbanion which is followed by protonation of this intermediate by Cys191. Our calculation showed that the carbanion-like intermediate transition state (INT2) at the $\mathrm{C}(3)$ position following the breaking of the $\mathrm{C}(2)-\mathrm{C}(3)$ bond is characterised by an activation energy of about $3 \mathrm{kcal} / \mathrm{mol}$ higher than the INT1. The proton transferred from Cys 191 thiol group to the intermediate with a $\Delta \mathrm{G}^{\ddagger}$ of $10.0 \mathrm{kcal} / \mathrm{mol}$ (TS3). From the crystal structure, the most probable candidate to play the role of the catalytic acid would be Cys191 and the water molecule bound to $\mathrm{Mg}^{2+}$ based on their $\mathrm{p} K_{\mathrm{a}}$ and distances (Supplementary Information Figure S1). In addition, the interatomic distance between Cys191 SH group and succinate $\mathrm{C}(3)$ atom in INT2 is $1.92 \AA$ compared to $3.64 \AA$ between succinate $\mathrm{C}(3)$ atom and W1. Likewise, either Asp108 or Arg228 were too far (>4 A) from succinate C(3) atom to donate a proton. Therefore, Cys191 would be the most suitable candidate to serve as a catalytic acid in the cleavage of isocitrate by ICL.

Alternative pathway II. As mentioned earlier, the experimental data showed that water molecules may shuttle proton from the hydroxyl group of the isocitrate $\mathrm{C}(2)$ atom via Asp108 or Glu182 ${ }^{15}$. Therefore, we also considered another scenario whereby the deprotonated atom was transferred to Glu182 (Fig. 2B). The QM/ $\mathrm{MM}$ calculations revealed that the transfer of a proton to Glu182 could occur but would require a higher activation energy $(\sim 26 \mathrm{kcal} / \mathrm{mol})$ compared to the transfer of proton to Asp108. The $\Delta \mathrm{G}^{\ddagger}$ for the intermediate step (INT3) is also higher compared to the protonation stage of Asp108, indicating that concerted proton transfer step in Glu182 is less favourable due to a higher energy barrier. The bond breaking step of isocitrate $\mathrm{C}(2)-\mathrm{C}(3)$ could be slower based on the interatomic distance compared to pathway I (TS2). The activation energy of the rate-determining step associated with $\mathrm{C}(2)-\mathrm{C}(3)$ bond breaking is characterized by a higher energy barrier of $35 \mathrm{kcal} / \mathrm{mol}$ compared to the pathway I. The formation of the products (glyoxylate and succinate) was also observed to have a high activation energy. In addition, the analysis of the entropy associated with the product formation step revealed that the products formed in pathway II possesses lower entropy compared to that in pathway I (Supplementary Information Table S1). Second order perturbation theory calculation to analyse the natural bond orbital was also performed to further study the stabilization energy and the intermolecular charge transfer associated with TS1 and TS4.

Natural bond orbital (NBO) analysis. NBO analysis from the second order perturbation theory calculation demonstrates that higher stabilization energy $\left(\mathrm{E}^{2}\right)$ values indicate stronger intermolecular interactions with a higher charge transfer between electron-donor and electron-acceptors due to hyperconjugation ${ }^{32-34}$. Table 2 shows that the stabilization energy $\mathrm{E}^{2}$ for the deprotonation of the isocitrate $\mathrm{C}(2)-\mathrm{OH}\left(\mathrm{O}_{68}\right)$ for pathway I represented as TS1 is $64.4 \mathrm{kcal} / \mathrm{mol}$, indicating more electron charge transfer from a lone pair $\mathrm{LP}\left(\mathrm{O}_{68}\right)$ to $\mathrm{O}_{85}$ of W1. For pathway II, the $\mathrm{E}^{2}$ value was lower compared to pathway I. A precise contrast between transition states TS1 involving the transfer of proton to Asp108 and TS4 (proton transfer to Glu182) revealed that the pathway I transition state is favoured with higher stabilization energy. A similar comparison has been shown in the literature for other catalytic mechanism involving enzymes ${ }^{21}$. This calculation demonstrates that higher intermolecular interaction and charge transfer takes place in pathway I, involving the transfer of proton to Asp108 (Fig. 6). 


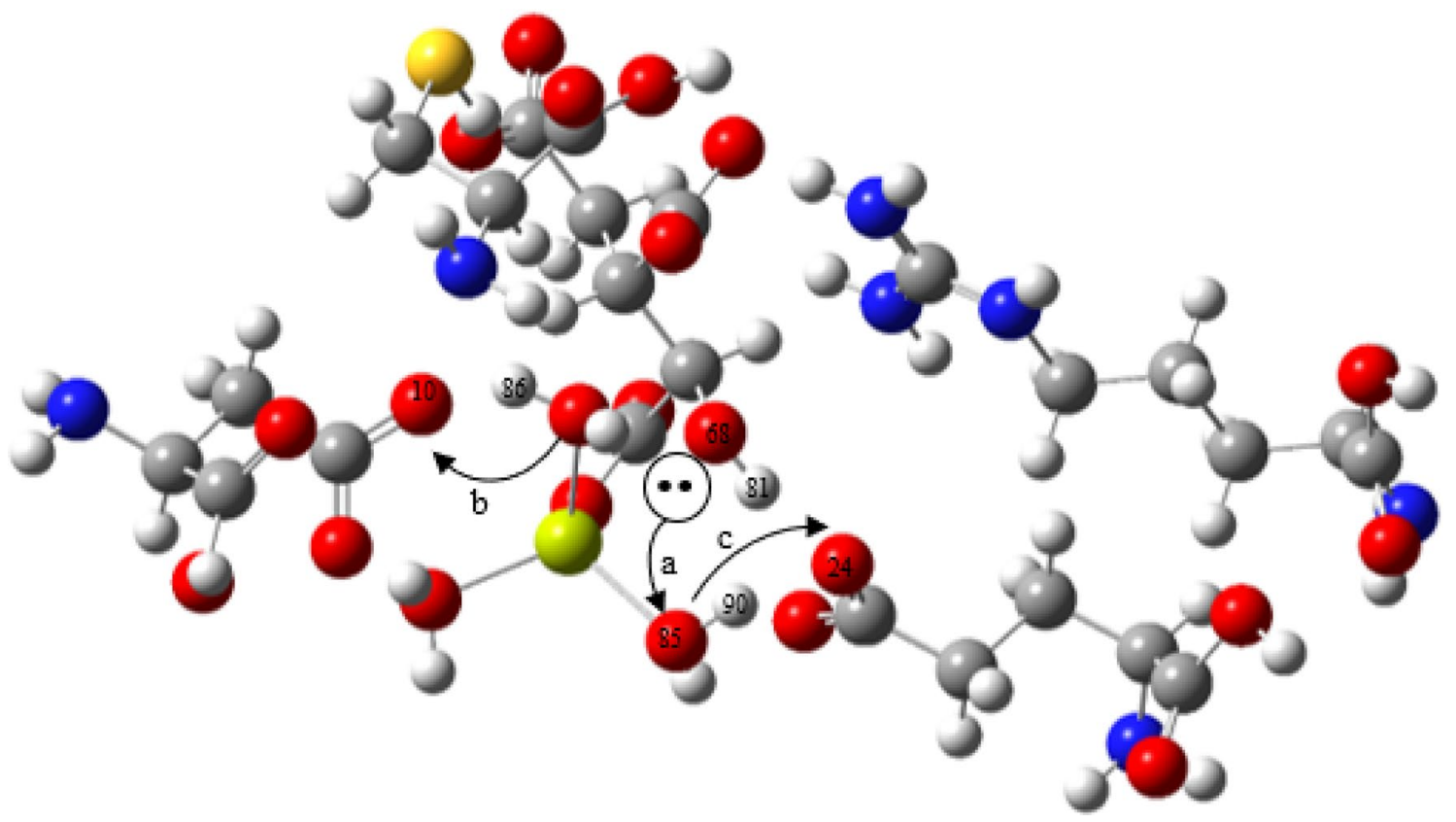

Figure 6. The depiction of electrons transfers for isocitrate-ICL complexes derived from second-order perturbation theory of NBO analysis. The curved arrows ( $a, b$ and c) illustrate the direction of charge transfer from lone pair to antibonding $\left(\mathrm{LP} \rightarrow \sigma^{\star}\right)$.

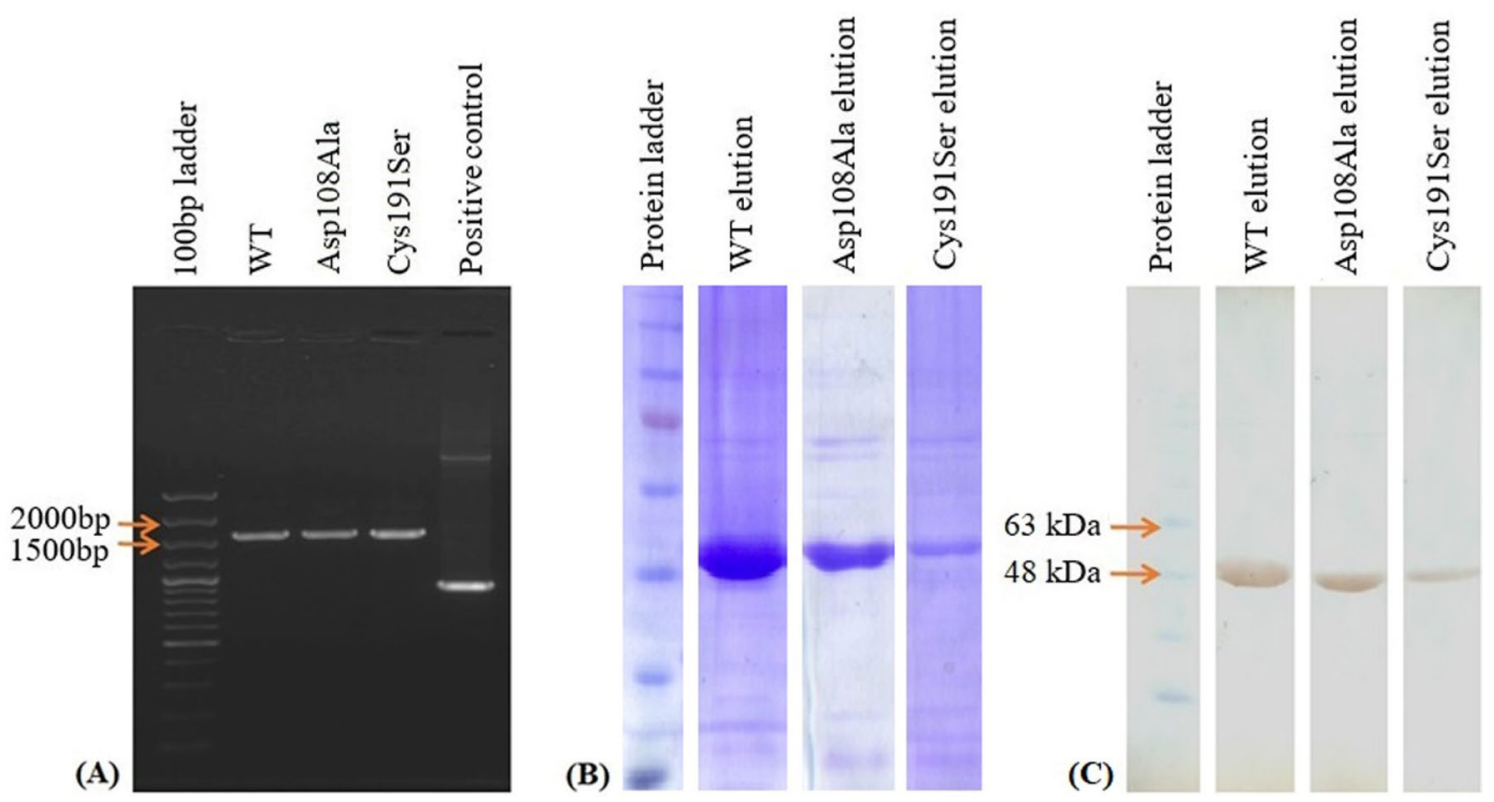

Figure 7. (A) The gel electrophoresis of colony PCR from wild type (WT), mutant type (MT) Asp108Ala and Cys191Ser M. tuberculosis isocitrate lyase (ICL) gene. The plasmid size for each sample is about $1600 \mathrm{bp}$. (B) The Coomasie Blue stained SDS-PAGE gel and (C) Western Blot of the purified WT, MT Asp108Ala and Cys191Ser M. tuberculosis ICL. The purified ICL is about $50 \mathrm{kDa}$. Full-length gels and blot are presented in Supplementary Information Figure S4.

M. tuberculosis ICL cloning, expression and purification. Extraction of pET28a plasmid containing the M. tuberculosis ICL gene was used due to the availability of the lac operon for inducible expression of the ICL protein. Site directed mutagenesis of the ICL was done to introduce point mutations at Asp108Ala and Cys191Ser. A total of 6 colonies from both mutated ICL plates were randomly picked for colony PCR. The amplified colony PCR product of the mutant (MT) and wild type (WT) ICL resolved at a correct DNA band size of $1600 \mathrm{bp}$ by electrophoresis (Fig. 7A). The sequencing results confirmed successful introduction of the desired point mutations in the ICL gene. The plasmids were then used for the expressions of the WT and MT ICL. The 


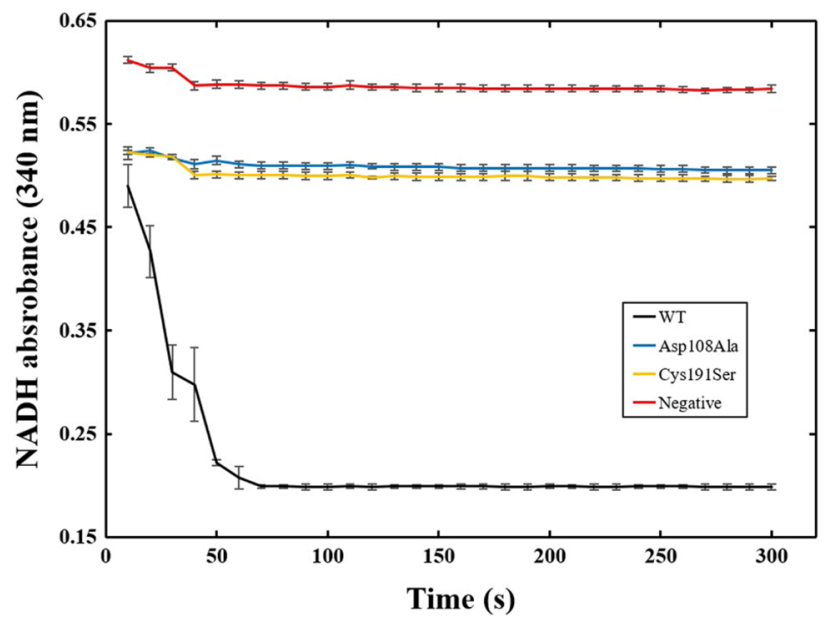

Figure 8. The NADH absorbance at $340 \mathrm{~nm}$ throughout $300 \mathrm{~s}$ reaction time for WT, MT Asp108Ala and Cys191Ser M. tuberculosis isocitrate lyase (ICL).

expressed ICL was extracted and purified from overnight culture using HIS-tagged purification method. Different elution and washing step were applied during the purification stage to ensure the proteins were purified to $\sim 95 \%$ purity. The purified ICL was tested on SDS-PAGE to confirm and validate the protein size from the expression. The WT and MT protein extracts have the same size of approximately $50 \mathrm{kDa}$ (Fig. 7B). To confirm the purified bands were indeed that of the ICL, Western Blotting analysis was carried out. The Western Blot analysis showed a distinct band at approximately $50 \mathrm{kDa}$ (Fig. 7C). From the gel and blot analysis, the yield of the Cys19Ser mutant appeared to be lower than the Asp108Ala mutant. The solubility of the WT protein provided a higher yield in comparison to the two mutants.

Enzymatic assay. Enzymatic assay of ICL was performed to validate and differentiate the functionality of the purified WT and MT ICL with isocitrate as the substrate. The ICL activity determination has been previously described $^{35}$. In general, an active ICL will cleave isocitrate into glyoxylate and succinate as the metabolites. With the presence of lactate dehydrogenase, NADH will be oxidised, followed by the reduction of glyoxylate to glycoxylate. The formation of glycoxylate will then reduce the concentration of NADH in the sample thus leading to a decreasing absorbance pattern of $\mathrm{NADH}$ which can be detected at $340 \mathrm{~nm}^{36}$. In this experiment, both MT ICL clones were considered inactive as the absorbance reading of NADH remained constant compared to WT ICL which underwent rapid absorbance decrease from $\sim 0.5$ to $\sim 0.2$ in $300 \mathrm{~s} \mathrm{(5} \mathrm{min)} \mathrm{reaction} \mathrm{time} \mathrm{(Fig.} \mathrm{8).}$

\section{Discussion}

The mechanistics involved in the cleavage of isocitrate is of interest due to its potential application for drug development. An earlier experiment proposed that water may act as a catalytic base since three water molecules labelled as W1, W2, W3 are coordinated to $\mathrm{Mg}^{2+}$ oriented towards the hydroxyl group attached to isocitrate $\mathrm{C}(2)$ atom $^{15}$. We observed through the energetics with lower activation energy (TS1) and a concerted process, water molecule W1 functioned as the catalytic base associated with the deprotonation of isocitrate $\mathrm{C}(2)-\mathrm{OH}$.

Our investigation also showed that the isocitrate cleavage mechanism follows a stepwise process and the results suggests that proton shuttling from the hydroxyl group of isocitrate $\mathrm{C}(2)$ is favourably transferred to Asp108 at a lower activation energy of $6.2 \mathrm{kcal} / \mathrm{mol}$ instead of Glu182. The rate-determining step of the reaction mechanism is associated with the breaking of the isocitrate $\mathrm{C}(2)-\mathrm{C}(3)$ bond followed by a proton transfer from Cys191, which was suggested as the catalytic acid. It was also observed that Arg228 or Asp108 was found to be far from the $\mathrm{C}(3)$ atom to donate a proton to the cleaved isocitrate, thus suggesting that Cys191 would be the most suitable candidate to serve as the catalytic acid in the cleavage of isocitrate by ICL. In this study, step 3 of the mechanism involves the breaking of isocitrate $\mathrm{C}(2)-\mathrm{C}(3)$ bond and it was observed that Arg228 plays an important role in this step by forming a strong bond with the carboxylate group which stabilizes the substrate. After the deprotonation of isocitrate C(2) hydroxyl group, Arg228 detaches from the substrates with a lengthened bond length making it difficult to contribute significantly to the break down of substrate.

The pathway II which involves the proton transfer from W1 to Glu182 was found to be with a high energy barrier making Glu182 unlikely to be involved in this catalytic mechanism. A further study of the entropy calculation showed that pathway I which involves water molecule W1 as the catalytic base had a more negative entropy compared to pathway II, indicating that pathway I mechanistic route is more favourable. Second order perturbation theory calculation was also performed to further study the stabilization energy and the intermolecular charge transfer associated with TS1 and TS4. The stabilization energy $E^{2}$ derived from second perturbation theory calculation also unveiled that TS1 of the pathway I had stronger intermolecular charge transfer compared to TS4 (pathway II). 
The theoretical calculation in this work was also supported by in vitro investigation. The Asp108Ala and Cys191Ser ICL mutants were non-reactive and did not generate the metabolites indicating the importance of Asp108 and Cys191 to the enzyme function. However, WT ICL remained active in the assay for the production of the metabolites. Therefore, the enzymatic assay further indicates the importance of both Asp108 and Cys191 to ensure the functionality and catalytic activity of $M$. tuberculosis ICL.

\section{Conclusion}

This study considered the catalytic mechanism for the cleavage of isocitrate by $M$. tuberculosis ICL using QM/ $\mathrm{MM}$ approach. We explored the ill-defined residues involved in the cleavage mechanism which was unclear from experimental findings. Our investigation showed that the cleavage mechanism follows a stepwise process and the proton shuttle from the hydroxyl group of the isocitrate $\mathrm{C}(2)$ atom is favourably transferred to Asp108 instead of Glu182 with a lower activation energy of $6.2 \mathrm{kcal} / \mathrm{mol}$. The rate-determining step of the reaction mechanism is the isocitrate $\mathrm{C}(2)-\mathrm{C}(3)$ bond break that is followed by a proton transfer from Cys191 which was suggested as the catalytic acid. The pathway II that involves the proton transfer from W1 to Glu182 was found to have a high energy barrier making Glu182 unlikely the residue to be involved in this catalytic mechanism. The stabilization energy $E^{2}$ derived from second perturbation theory calculation also unveiled that that TS1 of the pathway I had stronger intermolecular charge transfer compared to TS4 (pathway II). The revelation of the residues involved in $M$. tuberculosis ICL mechanism of inhibition could therefore provide better insights for future designs of new anti-tuberculosis drugs.

\section{Materials and methods}

Computational details. Structure preparations. The X-ray crystal structure of $M$. tuberculosis ICL with $\mathrm{PDB}$ id $1 \mathrm{~F}^{7} \mathrm{I}^{7}$ (glyoxylate and succinate as the ligands) and isocitrate with PDB id $1 \mathrm{XG}^{15}$ (MICL with isocitrate as the ligand) were aligned using Pymol ${ }^{37}$ program. The ligands of 1F81 (glyoxylate and succinate) and MICL of 1XG4 were then removed to generate the starting structure of isocitrate in complex with ICL. The Ser191 point mutation of ICL (in 1F81) was reverted to WT (Cys191). The three catalytic water molecules coordinated to $\mathrm{Mg}^{2+}$ in $1 \mathrm{~F} 8 \mathrm{I}$ was kept according to the possible catalytic mechanisms study ${ }^{38}$.

Molecular dynamics (MD) simulation. Molecular dynamics (MD) simulation was conducted using Amber $14^{39}$ with ff99SB force field ${ }^{40}$ for ICL and GAFF ${ }^{41}$ for isocitrate. The protonation states of ICL were assigned based on the $\mathrm{pK}_{\mathrm{a}}$ values computed by the empirical PropKa web server ${ }^{42}$. The complex was immersed in a $10 \AA$ TIP $3 \mathrm{P}^{43}$ cubic water box and $\mathrm{Na}^{+}$as counter ions were added to neutralize the system. Prior to MD simulation, two-stage geometric minimization was performed with 2500 steps of steepest decent followed with 2500 of conjugated gradient to relax steric clashes and closed contacts. The first stage minimization involved a constraint of $500 \mathrm{kcal} /$ $\mathrm{mol} / \AA^{2}$ while the second stage minimization did not involve any restraints. The system was then heated slowly to $300 \mathrm{~K}$ under the NVT ensemble for $500 \mathrm{ps}$ using the Langevin thermostat ${ }^{44}$ with a collision frequency of $1 \mathrm{ps}^{-1}$. A $10 \mathrm{~ns}$ of protein-retrained equilibration MD with force constraint of $10 \mathrm{kcal} / \mathrm{mol} / \AA^{2}$ was first performed. This was followed by $25 \mathrm{~ns}$ MD production run with 2 fs time step without harmonic restraints in the periodic boundary condition under the NPT ensemble, $300 \mathrm{~K}$ and $1 \mathrm{~atm}$. Partial Mesh Ewald (PME) algorithm ${ }^{45}$ was $^{2}$ employed to calculate long range electrostatic interactions while $12 \AA$ cutoff was set for van der Waals force. All bonds involving hydrogen atoms were constrained using SHAKE algorithm ${ }^{46}$. Analysis of RMSD was performed on ICL backbone $\mathrm{C}_{\alpha}$ atoms.

ONIOM QM/MM setup. The starting structure for QM/MM calculation was obtained for the last snapshot (with RMSD of $1.8 \AA$ compared to the crystal structure) of the $25 \mathrm{~ns}$ MD production run. Two-step minimizations were performed as described in the MD simulation to remove unfitting distortions. Truncation of the system was performed by removing water molecules beyond $15 \AA$ from isocitrate. A two-layered ONIOM method ${ }^{47-49}$ in Gaussian $09^{50}$ that was widely used in enzymatic systems ${ }^{21,51-53}$ was applied in this study. The ONIOM QMM/ MM system was divided into two layers: the high layer (QM) and the low layer (MM) (Fig. 5). The QM region consisted of 102 atoms and 6 link hydrogen atoms employed to saturate dangling bonds and to treat the boundary between $\mathrm{QM} / \mathrm{MM}$ region, while the rest was included in the MM region. To circumvent spurious deviations and fluctuations in the geometries, the QM/MM regions, residues and water molecules within $6 \AA$ around the active site were fully optimized while others were held fixed (2443 atoms in the MM region). Partial charges for the high layer was calculated using the restrained electrostatic potential (RESP) charge fitting ${ }^{54}$. To obtain a better understanding of the proposed reaction mechanism, potential energy surface (PES) for bond breaking (C2-C3) and deprotonation of the $\mathrm{C}(2)-\mathrm{OH}$ group reaction coordinate scan were explored using ONIOM implemented in Gaussian 09. In order to obtain a better understanding of the proposed reaction mechanism, potential energy surface (PES) scan was explored using ONIOM implemented in Gaussian 09. The PES scan is a two dimensional single scan which involves the $\mathrm{C}(2)-\mathrm{C}(3)$ and the deprotonation of $\mathrm{C}(2)-\mathrm{OH}$ group. When two coordinate scan is done simultaneously, it is called a $2 \mathrm{D}$ scan. The scan was performed prior to the final optimization and the starting structures for the transition state (TS) was obtained from the 2D scan (Fig. 4). Density functional theory (DFT) was applied in for QM optimization. M06-2X functional ${ }^{16,55}$ in conjunction with 6-31+G(d,p) basis set was used to obtain minima and transition states optimized geometries. The electrostatic interaction between the QM and MM region was described by the electronic embedding scheme ${ }^{51,56}$ implemented in ONIOM. The vibrational frequency was calculated to confirm that all transition states have only one negative frequency corresponding to the bond forming/breaking process and the minimum reaction energy path was determined by intrinsic reaction coordinate (IRC) calculation by ONIOM (M06-2X/6-31+G(d,p): AMBER) level. Single point calculation with electronic embedding scheme ${ }^{57}$ at ONIOM (M06-2X/6-31+G(d,p): AMBER) was performed 
on the obtained optimized structures (reactant, transition state and product). Two functionals- mPWB1W/6$311++\mathrm{G}(2 \mathrm{~d}, 2 \mathrm{p})$ and $\omega \mathrm{b} 97 \mathrm{XD} / 6-311++\mathrm{G}(2 \mathrm{~d}, 2 \mathrm{p})$ were subsequently used for single point calculations to further evaluate the sensitivity of the density functional. These functionals have been reported to give accurate performances for both thermodynamics and kinetics when used together with the large 6-311++G(2d,2p) basis set ${ }^{31,58}$.

Natural bond orbital (NBO) calculations. Second-order perturbation analysis was performed on the transition states (TS) structures (TS1 and TS4). In NBO analysis, high $\mathrm{E}^{2}$ value demonstrates robust and strong interaction between acceptor and donors in a molecular system. The electronic wave functions are construed in terms of a set of unoccupied non-Lewis localised and molecular orbitals. The conjugative (delocalisation) interaction was determined using Eq. (1):

$$
E^{2}=\Delta E_{i j}=q_{j} \frac{F(i, j)^{2}}{\varepsilon_{j}-\varepsilon_{i}}
$$

where $q_{j}$ is the donor orbital occupancy, $\varepsilon_{i}$ and $\varepsilon_{j}$ are diagonal matrix elements and $F(i, j)$ is the off-diagonal Fock matrix element.

Laboratory experimental details. Materials. DH5a and DreamTaq DNA polymerase from Thermo Fisher Scientific; designed primers (T7 promoter and T7 terminator) were synthesized by IDT DNA Technologies; Vent DNA polymerase from New England Biolabs; Ni-NTA agarose, plasmid extraction and Qiaprep Miniprep kit (Qiagen); Escherichia coli BL21, lactate dehydrogenase, nicotinamide adenine dinucleotide (NADH), MOPs, $\mathrm{MgCl}_{2}$, L-cysteine, ethylenediaminetetraacetic acid (EDTA), isocitrate from Sigma Aldrich; IPTG from OmniPur; lysozyme from Norgen Biotek; 2YT media (Novagen); skim milk, kanamycin and peroxidase stain DAB kit from Nacalai Tesque; goat anti-mouse HRP from Dako; nitrocellulose membrane from BioTrace; anti6-His epitope tag from BioLegend.

Site directed mutagenesis of M. tuberculosis ICL. Wild type (WT) M. tuberculosis ICL gene was PCR-amplified with the designed primers (Supplementary Information Table S2) to produce Asp108Ala and Cys191Ser mutants. PCR reaction mix $(20 \mu \mathrm{l})$ containing $2 \mu \mathrm{l}$ Vent buffer, $1 \mu \mathrm{l}$ each for forward and reverse primer, $2 \mu \mathrm{l}$ dNTPs, $0.2 \mu$ l Vent DNA polymerase, $1 \mu$ l purified plasmid PM107 and $12.8 \mu$ l distilled water. PCR amplification condition was set up with $3 \mathrm{~min}\left(95^{\circ} \mathrm{C}\right)$ initial denaturation, $30 \mathrm{~s}\left(95^{\circ} \mathrm{C}\right)$ denaturation, $1 \mathrm{~min}\left(55^{\circ} \mathrm{C}\right)$ annealing, $7 \mathrm{~min}\left(72^{\circ} \mathrm{C}\right)$ elongation for 30 cycles and ended with $15 \mathrm{~min}\left(72^{\circ} \mathrm{C}\right)$ as final extension. The PCR products were digested with $1 \mu \mathrm{l}$ DpnI restriction enzyme to breakdown the original template. The digested PCR product was then transformed into DH5a via electroporation. The transformants were screened with colony PCR and the DNA sequence was checked to verify the desired mutants were acquired.

Expression of $M$. tuberculosis ICL. The purified plasmids of the verified clones were transformed into expression vector $E$. coli BL21. Cells containing recombinant constructs were inoculated into $5 \mathrm{ml} 2 \mathrm{YT}$ media with kanamycin and grown overnight at $37^{\circ} \mathrm{C}$. The culture was diluted 1:100 with fresh 2YT media containing kanamycin and cultured at $37^{\circ} \mathrm{C}, 200 \mathrm{rpm}$ until $\mathrm{OD}_{600 \mathrm{~nm}}$ reached $0.6 \mathrm{~nm}$. Induction was initiated with $40 \mu \mathrm{M} 1 \mathrm{M}$ IPTG and incubation continued at $25^{\circ} \mathrm{C}, 160 \mathrm{rpm}$ overnight.

Extraction and purification of $M$. tuberculosis ICL. The overnight culture was centrifuged at $8000 \mathrm{rpm}, 12^{\circ} \mathrm{C}$ for $10 \mathrm{~min}$. The bacterial pellet was re-suspended with $3 \mathrm{ml}$ lysis buffer $\left[50 \mathrm{mM} \mathrm{NaH}_{2} \mathrm{PO}_{4}, 500 \mathrm{mM} \mathrm{NaCl}, 20 \mu \mathrm{g} / \mathrm{ml}\right.$ lysozyme, $\mathrm{pH}$ 8]. The cell lysate was sonicated (Sonicator 3000, Misonix) with power of 3.5 for $3 \mathrm{~min}$, followed by centrifugation at $8000 \mathrm{rpm}, 12^{\circ} \mathrm{C}$ for $30 \mathrm{~min}$. The supernatant with ICL was purified with Ni-NTA agarose affinity chromatography column, with increasing imidazole concentration for elution.

SDS-PAGE and western blot analysis of M. tuberculosis ICL. Elution fractions containing expressed target protein were identified with SDS-PAGE and Western Blot. In SDS-PAGE analysis, the sample aliquots were mixed with $2 \times$ Laemmli sample buffer and electrophoresed in 10\% SDS-polyacrylamide gel. Resulting protein bands were visualized with Coomasie Blue stain. For Western Blot, the protein from the electrophoresed gel was transferred to nitrocellulose membrane. The membrane was initially blocked with $2 \%$ skim milk for $1 \mathrm{~h}$, followed by $1 \mathrm{~h}$ incubation with anti-6-His epitope tag and $1 \mathrm{~h}$ incubation with goat anti-mouse HRP. In between incubation steps were the washing steps with $1 \times$ phosphate-buffered saline (PBS) with Tween 20 (PBS-T) 5 min for 3 times. The antibody tagged protein were visualized with peroxidase stain DAB kit.

M. tuberculosis ICL enzymatic assay. The reaction mixture was prepared with $58.3 \mu$ purified ICL, $2.1 \mu \mathrm{LDH}$, $46.7 \mu \mathrm{l} \mathrm{NADH}$ and MOPS buffer to a final volume of $700 \mu \mathrm{l}$. The mixture was transferred into a microtitre plate and pre-incubated at $37^{\circ} \mathrm{C}$, for $5 \mathrm{~min}$ followed by addition of $100 \mu \mathrm{l} 20 \mathrm{mM}$ isocitrate. The absorbance was immediately checked at $340 \mathrm{~nm}$ for $300 \mathrm{~s}$ at $10 \mathrm{~s}$ interval time. A negative control was set without the addition of purified ICL into the reaction mixture. Equations (2) and (3) are the calculations for ICL activity and specific activity, respectively. The calculated $\mathrm{K}_{\mathrm{m}}$ and $\mathrm{V}_{\max }$ are $25 \mathrm{mM}$ and $0.97 \mu \mathrm{mol} / \mathrm{min} / \mathrm{mg}$, respectively.

$$
\text { Enzyme activity }(\mathrm{U} / \mathrm{ml})=\frac{(\text { absornace } / \text { min })(\text { total volume })(D F)}{(\text { NADH extinction coefficient })(\text { sample volume })(\text { light path })}
$$




$$
\text { Specific activity }(\mu \mathrm{mol} / \mathrm{min} / \mathrm{mg})=\frac{\text { Enzyme activity }}{\text { ICL concentration }}
$$

Received: 8 April 2020; Accepted: 7 October 2020

Published online: 03 November 2020

\section{References}

1. Lu, Z. et al. Diversity of function in the isocitrate lyase enzyme superfamily: the Dianthus caryophyllus petal death protein cleaves $\alpha$-keto and a-hydroxycarboxylic acids. Biochemistry 44, 16365-16376. https://doi.org/10.1021/bi0517761 (2005).

2. Narayanan, B. C. et al. Structure and function of PA4872 from Pseudomonas aeruginosa, a novel class of oxaloacetate decarboxylase from the PEP mutase/isocitrate lyase superfamily. Biochemistry 47, 167-182. https://doi.org/10.1021/bi701954p (2008).

3. Dunn, M. F., Ramirez-Trujillo, J. A. \& Hernández-Lucas, I. Major roles of isocitrate lyase and malate synthase in bacterial and fungal pathogenesis. Microbiology 155, 3166-3175. https://doi.org/10.1099/mic.0.030858-0 (2009).

4. Betts, J. C., Lukey, P. T., Robb, L. C., McAdam, R. A. \& Duncan, K. Evaluation of a nutrient starvation model of Mycobacterium tuberculosis persistence by gene and protein expression profiling. Mol. Microbiol. 43, 717-731. https://doi.org/10.104 6/j.1365-2958.2002.02779.x (2002).

5. Muñoz-Elías, E. J. \& McKinney, J. D. Mycobacterium tuberculosis isocitrate lyases 1 and 2 are jointly required for in vivo growth and virulence. Nat. Med. 11, 638-644. https://doi.org/10.1038/nm1252 (2005).

6. Gould, T. A., Van De Langemheen, H., Muñoz-Elías, E. J., McKinney, J. D. \& Sacchettini, J. C. Dual role of isocitrate lyase 1 in the glyoxylate and methylcitrate cycles in Mycobacterium tuberculosis. Mol. Microbiol. 61, 940-947. https://doi.org/10.111 $1 / j .1365-2958.2006 .05297 . x(2006)$.

7. Sharma, V. et al. Structure of isocitrate lyase, a persistence factor of Mycobacterium tuberculosis. Nat. Struct. Mol. Biol. 7, 663. https ://doi.org/10.1038/77964 (2000).

8. Lee, Y. V., Choi, S. B., Wahab, H. A. \& Choong, Y. S. Active site flexibility of Mycobacterium tuberculosis isocitrate lyase in dimer form. J. Chem. Inf. Model. 57, 2351-2357. https://doi.org/10.1021/acs.jcim.7b00265 (2017).

9. Shukla, H. et al. Insight into the structural flexibility and function of Mycobacteriumtuberculosis isocitrate lyase. Biochimie 110, 73-80. https://doi.org/10.1016/j.biochi.2014.12.016 (2015).

10. Shukla, H. et al. Salt-regulated reversible fibrillation of Mycobacterium tuberculosis isocitrate lyase: Concurrent restoration of structure and activity. Int. J. Biol. Macromol. 104, 89-96. https://doi.org/10.1016/j.ijbiomac.2017.06.008 (2017).

11. Shukla, R., Shukla, H. \& Tripathi, T. Activity loss by H46A mutation in Mycobacterium tuberculosis isocitrate lyase is due to decrease in structural plasticity and collective motions of the active site. Tuberculosis (Edinb) 108, 143-150. https://doi.org/10.1016/j. tube.2017.11.013 (2018).

12. Lee, Y. V., Choi, S. B., Wahab, H. A., Lim, T. S. \& Choong, Y. S. Applications of ensemble docking in potential inhibitor screening for Mycobacterium tuberculosis isocitrate lyase using a local plant database. J. Chem. Inf. Model. 59, 2487-2495. https://doi. org/10.1021/acs.jcim.8b00963 (2019).

13. Shukla, H. et al. Isocitrate lyase of Mycobacterium tuberculosis is inhibited by quercetin through binding at $\mathrm{N}$-terminus. Int. J. Biol. Macromol. 78, 137-141. https://doi.org/10.1016/j.ijbiomac.2015.04.005 (2015).

14. Shukla, R., Shukla, H., Sonkar, A., Pandey, T. \& Tripathi, T. Structure-based screening and molecular dynamics simulations offer novel natural compounds as potential inhibitors of Mycobacterium tuberculosis isocitrate lyase. J. Biomol. Struct. Dyn. 36, 2045-2057. https://doi.org/10.1080/07391102.2017.1341337 (2018)

15. Liu, S. et al. Crystal structures of 2-methylisocitrate lyase in complex with product and with isocitrate inhibitor provide insight into lyase substrate specificity, catalysis and evolution. Biochemistry 44, 2949-2962. https://doi.org/10.1021/bi0479712 (2005).

16. Jongkon, N., Chotpatiwetchkul, W. \& Gleeson, M. P. Probing the catalytic mechanism involved in the isocitrate lyase superfamily: hybrid quantum mechanical/molecular mechanical calculations on 2, 3-dimethylmalate lyase. J. Phys. Chem. B 119, 11473-11484. https://doi.org/10.1021/acs.jpcb.5b04732 (2015).

17. King, D. T., Worrall, L. J., Gruninger, R. \& Strynadka, N. C. J. New Delhi metallo- $\beta$-lactamase: Structural insights into $\beta$-lactam recognition and inhibition. J. Am. Chem. Soc. 134, 11362-11365. https://doi.org/10.1021/ja303579d (2012).

18. Zhang, H. \& Hao, Q. Crystal structure of NDM-1 reveals a common $\beta$-lactam hydrolysis mechanism. FASEB J. 25, $2574-2582$. https://doi.org/10.1096/fi.11-184036 (2011).

19. Narayanan, B. et al. Structure and function of 2,3-dimethylmalate lyase, a PEP mutase/isocitrate lyase superfamily member. J. Mol. Biol. 386, 486-503. https://doi.org/10.1016/j.jmb.2008.12.037 (2009).

20. Bhusal, R. P., Bashiri, G., Kwai, B. X., Sperry, J. \& Leung, I. K. Targeting isocitrate lyase for the treatment of latent tuberculosis. Drug Discov. Today 22, 1008-1016 (2017).

21. Ibeji, C. U. et al. The catalytic role of water in the binding site of L, D-Transpeptidase 2 within acylation mechanism: a QM/MM (ONIOM) modeling. Tuberculosis 113, 222-230. https://doi.org/10.1016/j.tube.2018.10.005 (2018).

22. Singh, W., Quinn, D., Moody, T. S. \& Huang, M. Reaction mechanism of histone demethylation in $\alpha$ KG-dependent non-heme iron enzymes. J. Phys. Chem. B 123, 7801-7811. https://doi.org/10.1021/acs.jpcb.9b06064 (2019).

23. Tolufashe, G. F. et al. Structure and function of L, D-and D, D-transpeptidase family enzymes from Mycobacterium tuberculosis. Curr. Med. Chem. https://doi.org/10.2174/0929867326666181203150231 (2018).

24. Zhang, J. \& Liu, Y. A QM/MM study of the catalytic mechanism of aspartate ammonia lyase. J. Mol. Graph. Model. 51, 113-119. https://doi.org/10.1016/j.jmgm.2014.05.003 (2014).

25. Daniels, A. D. et al. Reaction mechanism of $\mathrm{N}$-acetylneuraminic acid lyase revealed by a combination of crystallography, QM/ MM simulation, and mutagenesis. ACS Chem. Biol. 9, 1025-1032. https://doi.org/10.1021/cb500067z (2014).

26. Su, H., Dong, L. \& Liu, Y. A QM/MM study of the catalytic mechanism of a-1,4-glucan lyase from the red seaweed Gracilariopsis lemaneiformis. RSC Adv. 4, 54398-54408. https://doi.org/10.1039/C4RA09758K (2014).

27. Zhu, W., Liu, Y. \& Zhang, R. A QM/MM study of the reaction mechanism of (R)-hydroxynitrile lyases from Arabidopsisthaliana (AtHNL). Proteins Struct. Funct. Bioinform. 83, 66-77 (2015).

28. Schlippe, Y. V. G. \& Hedstrom, L. A twisted base? The role of arginine in enzyme-catalyzed proton abstractions. Arch. Biochem. Biophys. 433, 266-278. https://doi.org/10.1016/j.abb.2004.09.018 (2005).

29. van der Kamp, M. W., Perruccio, F. \& Mulholland, A. J. High-level QM/MM modelling predicts an arginine as the acid in the condensation reaction catalysed by citrate synthase. Chem. Commun. https://doi.org/10.1039/b800496j (2008).

30. Condic-Jurkic, K., Zipse, H. \& Smith, D. M. A compound QM/MM procedure: comparative performance on a pyruvate formatelyase model system. J. Comput. Chem. 31, 1024-1035. https://doi.org/10.1002/jcc.21389 (2010).

31. Calixto, A. R., Brás, N. F., Fernandes, P. A. \& Ramos, M. J. Reaction mechanism of human renin studied by quantum mechanics/ molecular mechanics (QM/MM) calculations. ACS Catal. 4, 3869-3876. https://doi.org/10.1021/cs500497f (2014).

32. Arnaud, R. Ab initio study of some $\mathrm{CH}_{3} \mathrm{OCXYCH}$ radicals: the influence of anomeric effects on their structure and their stability. J. Comput. Chem. 15, 1341-1356. https://doi.org/10.1002/jcc.540151204 (1994). 
33. Kwon, O., Sevin, F. \& MlL, McKee. Density functional calculations of methyllithium, t-butyllithium, and phenyllithium oligomers: effect of hyperconjugation on conformation. J. Phys. Chem. A 105, 913-922. https://doi.org/10.1021/jp003345c (2001).

34. Rauf, S. M. A. et al. The effect of N-methylation of amino acids (Ac-X-OMe) on solubility and conformation: a DFT study. Org. Biomol. Chem. 13, 9993-10006. https://doi.org/10.1039/C5OB01565K (2015).

35. Höner Zu Bentrup, K., Miczak, A., Swenson, D. L. \& Russell, D. G. Characterization of activity and expression of isocitrate lyase in Mycobacterium avium and Mycobacterium tuberculosis. J. Bacteriol. 181, 7161-7167. https://doi.org/10.1128/jb.181.23.71617167.1999 (1999).

36. Yin, Y. H. et al. Screening peptide inhibitors using phage peptide library with isocitrate lyase in Mycobacterium tuberculosis as target. Chem. Res. Chin. Univ. 27, 635-640 (2011).

37. DeLano, W. L. PyMOL: an open-source molecular graphics tool. CCP4 Newslett. Protein Crystallogr. 40, 82-92 (2002).

38. Cao, Y., Han, S., Yu, L., Qian, H. \& Chen, J. Z. MD and QM/MM studies on long-chain L- $\alpha$-hydroxy acid oxidase: substrate binding features and oxidation mechanism. J. Phys. Chem. B 118, 5406-5417. https://doi.org/10.1021/jp5022399 (2014).

39. Case, D. A. et al. AMBER 14 (University of California, San Francisco, 2014).

40. Hornak, V. et al. Comparison of multiple Amber force fields and development of improved protein backbone parameters. Proteins Struct. Funct. Bioinform. 65, 712-725. https://doi.org/10.1002/prot.21123 (2006).

41. Wang, J., Wolf, R. M., Caldwell, J. W., Kollman, P. A. \& Case, D. A. Development and testing of a general amber force field. J. Comput. Chem. 25, 1157-1174. https://doi.org/10.1002/jcc.20035 (2004).

42. Li, H., Robertson, A. D. \& Jensen, J. H. Very fast empirical prediction and rationalization of protein $\mathrm{pK}_{\mathrm{a}}$ values. Proteins Struct. Funct. Bioinform. 61, 704-721. https://doi.org/10.1002/prot.20660 (2005).

43. Jorgensen, W. L., Chandrasekhar, J., Madura, J. D., Impey, R. W. \& Klein, M. L. Comparison of simple potential functions for simulating liquid water. J. Chem. Phys. 79, 926-935. https://doi.org/10.1063/1.445869 (1983).

44. Izaguirre, J. A., Catarello, D. P., Wozniak, M. \& Skeel, R. D. Langevin stabilization of molecular dynamics. J. Chem. Phys. 114, 2090-2098. https://doi.org/10.1063/1.1332996 (2001).

45. Harvey, M. J. \& De Fabritiis, G. An implementation of the smooth particle mesh Ewald method on GPU hardware. J. Chem. Theory Comput. 5, 2371-2377. https://doi.org/10.1021/ct900275y (2009).

46. Ryckaert, J. P., Ciccotti, G. \& Berendsen, H. J. C. Numerical integration of the cartesian equations of motion of a system with constraints: molecular dynamics of $n$-alkanes. J. Comput. Phys. 23, 327-341. https://doi.org/10.1016/0021-9991(77)90098-5 (1977).

47. Svensson, M. et al. ONIOM: a multilayered integrated MO+ MM method for geometry optimizations and single point energy predictions. A test for Diels-Alder reactions and $\mathrm{Pt}\left(\mathrm{P}(\mathrm{t}-\mathrm{Bu})_{3}\right)_{2}+\mathrm{H}_{2}$ oxidative addition. J. Phys. Chem. 100, 19357-19363. https ://doi.org/10.1021/jp962071j (1996).

48. Vreven, T., Morokuma, K., Farkas, Ö., Schlegel, H. B. \& Frisch, M. J. Geometry optimization with QM/MM, ONIOM, and other combined methods. I. Microiterations and constraints. J. Comput. Chem. 24, 760-769 (2003).

49. Suresh, C. H., Vargheese, A. M., Vijayalakshmi, K. P., Mohan, N. \& Koga, N. Role of structural water molecule in HIV proteaseinhibitor complexes: a QM/MM study. J. Comput. Chem. 29, 1840-1849. https://doi.org/10.1002/jcc.20961 (2008).

50. Gaussion 09, Revision D. 01 (Gaussian Inc., Wallingford, 2013).

51. Lu, S. Y., Jiang, Y. J., Lv, J., Zou, J. W. \& Wu, T. X. Role of bridging water molecules in GSK3beta-inhibitor complexes: insights from QM/MM, MD, and molecular docking studies. J. Comput. Chem. 32, 1907-1918. https://doi.org/10.1002/jcc.21775 (2011).

52. Shi, R., Li, W. J., Liu, G. \& Tang, Y. Catalytic mechanism of cytochrome P450 2D6 for 4-hydroxylation of aripiprazole: a QM/MM study. Chin. J. Chem. 31, 1219-1227. https://doi.org/10.1002/cjoc.201300427 (2013).

53. McClory, J., Timson, D. J., Singh, W., Zhang, J. \& Huang, M. Reaction mechanism of isopentenyl phosphate kinase: a QM/MM study. J. Phys. Chem. B 121, 11062-11071. https://doi.org/10.1021/acs.jpcb.7b08770 (2017).

54. Bayly, C. I., Cieplak, P., Cornell, W. \& Kollman, P. A. A well-behaved electrostatic potential based method using charge restraints for deriving atomic charges: the RESP model. J. Phys. Chem. 97, 10269-10280. https://doi.org/10.1021/j100142a004 (1993).

55. Zhao, Y. \& Truhlar, D. G. The M06 suite of density functionals for main group thermochemistry, thermochemical kinetics, noncovalent interactions, excited states, and transition elements: two new functionals and systematic testing of four M06-class functionals and 12 other functionals. Theor. Chem. Acc. 120, 215-241 (2008).

56. Zhou, J. et al. QM/MM studies of the matrix metalloproteinase 2 (MMP2) inhibition mechanism of (S)-SB-3CT and its oxirane analogue. J. Chem. Theory Comput. 6, 3580-3587. https://doi.org/10.1021/ct100382k (2010).

57. Vreven, T. et al. Combining quantum mechanics methods with molecular mechanics methods in ONIOM. J. Chem. Theory Comput. 2, 815-826. https://doi.org/10.1021/ct050289g (2006).

58. Ibeji, C. U. et al. The driving force for the acylation of $\beta$-lactam antibiotics by L, D-transpeptidase 2: quantum mechanics/molecular mechanics (QM/MM) study. ChemPhysChem 20, 1126-1134 (2019).

\section{Acknowledgements}

This work was supported by Fundamental Research Grant Scheme (FRGS; 203/CIPPM/6711680) from Malaysia Ministry of Education. C.U.I. is also thankful to CHPC (https://www.chpc.ac.za) for computational resources.

\section{Author contributions}

Y.S.C. designed the research project. C.U.I. performed computational experiments, collected and analyzed the data. N.A.M.S., J.S.S and A.C.W.C. performed in vitro experiments, collected and analyzed the data. C.U.I., J.S.S, T.S.L. and Y.S.C. wrote the paper.

\section{Competing interests}

The authors declare no competing interests.

\section{Additional information}

Supplementary information is available for this paper at https://doi.org/10.1038/s41598-020-75799-8.

Correspondence and requests for materials should be addressed to Y.S.C.

Reprints and permissions information is available at www.nature.com/reprints.

Publisher's note Springer Nature remains neutral with regard to jurisdictional claims in published maps and institutional affiliations. 
(c) (i) Open Access This article is licensed under a Creative Commons Attribution 4.0 International cc) License, which permits use, sharing, adaptation, distribution and reproduction in any medium or format, as long as you give appropriate credit to the original author(s) and the source, provide a link to the Creative Commons licence, and indicate if changes were made. The images or other third party material in this article are included in the article's Creative Commons licence, unless indicated otherwise in a credit line to the material. If material is not included in the article's Creative Commons licence and your intended use is not permitted by statutory regulation or exceeds the permitted use, you will need to obtain permission directly from the copyright holder. To view a copy of this licence, visit http://creativecommons.org/licenses/by/4.0/.

(C) The Author(s) 2020 